\title{
A PRACTICAL METHOD FOR MEASURING THE SPATIAL FREQUENCY RESPONSE OF LIGHT FIELD CAMERAS
}

\author{
Damien Firmenich, Lö̈c Baboulaz, Sabine Süsstrunk \\ School of Computer and Communication Sciences, \\ Ecole Polytechnique Fédérale de Lausanne \\ \{damien.firmenich, loic.baboulaz, sabine.susstrunk\}@epfl.ch
}

\begin{abstract}
The spatial frequency response (SFR) is one of the most important and unbiased image quality measures of a digital camera. It evaluates to which extent a lens/sensor combination can resolve scene details. In this paper, we propose a simple and practical method to measure the SFR of microlensbased light field cameras. The particularity of such cameras resides in their ability to capture both spatial and angular information of the incoming light field thanks to an array of microlenses located in front of the sensor. Existing methods for measuring the SFR of conventional cameras are thus no longer applicable as the interaction between the main lens and the micro-lenses results in different resolving powers over the image plane that depend on the scene depths. By using a 3-dimensional target made of vertical lines printed on an inclined planar surface, we are able to measure the SFR across multiple depths in a single exposure. Our method allows SFR measurements from the raw light field itself as captured by the camera, and is thus independent of subsequent post-processing algorithms such as image reconstruction, digital refocusing or depth estimation. Our experimental results are consistent with theoretical bounds and reproducible.
\end{abstract}

Index Terms - Spatial frequency response, Modulation transfer function, Plenoptic cameras, Light field, Computational photography

\section{INTRODUCTION}

Light field cameras, such as the ones developed by Lytro [1], are able to capture a 4D light field from a single exposure. This is achieved by inserting a microlens array between the sensor and the main lens. Each microlens projects a low resolution microimage on the sensor, which contains directional samples for a single spatial location. From this information, it is possible to retrace the light rays in space and develop new post-processing applications such as single exposure digital refocusing or depth estimation.

The spatial sampling is determined by the microlens array whereas the angular sampling is determined by the pixels on the sensor. This implies a trade-off between spatial and angular resolution as the total number of captured light rays is

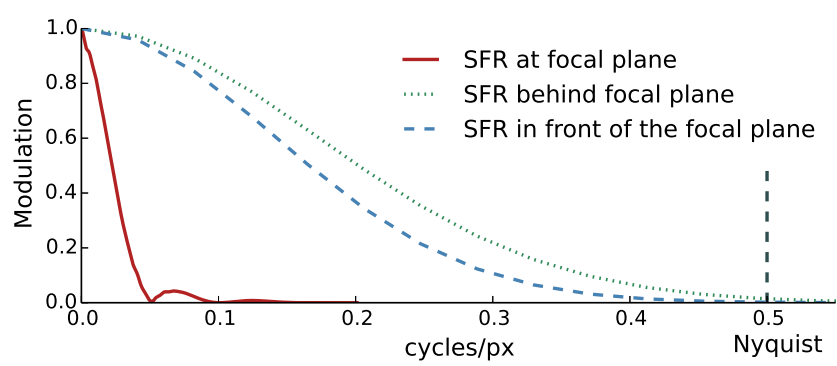

Fig. 1: The spatial frequency responses (SFRs) of a microlens-based light field camera. The SFR varies according to scene depth, and is lowest at the focal plane.

limited by the sensor resolution. In the case of first generation Lytro camera, the sensor has a resolution of $3280 \times 3280$ pixels, with $330 \times 330$ microlenses arranged in a hexagonal lattice. The microimages thus have a resolution of approximately $10 \times 10$ pixels.

Given the early stage of development of light field cameras, not many methods have yet been proposed to assess their intrinsic quality using an objective criterion such as their spatial frequency response (SFR). The SFR, analogue to the modulation transfer function of an optical system, is a resolution measure that reports how well a lens/sensor combination is able to resolve scene details. The SFR describes the modulation at different spatial frequencies, usually expressed in cycles/pixel. The modulation expresses how well the original contrast of the target is reproduced. When the spatial frequencies are normalized, as illustrated in Fig. 1, the Nyquist limit is at 0.5 cycles/pixel.

For conventional cameras, SFR measurement standards exist such as ISO12233 [2], which define a planar reference target and an evaluation method. These methods are not only used to assess the quality of a given camera, but to also objectively compare different lens/sensor combinations among each other. They all evaluate the SFR from the acquired raw image, before any post-processing is applied, in order to avoid any modification of the modulation that would be induced by processing the data.

Light field cameras, however, have two main optical parts 

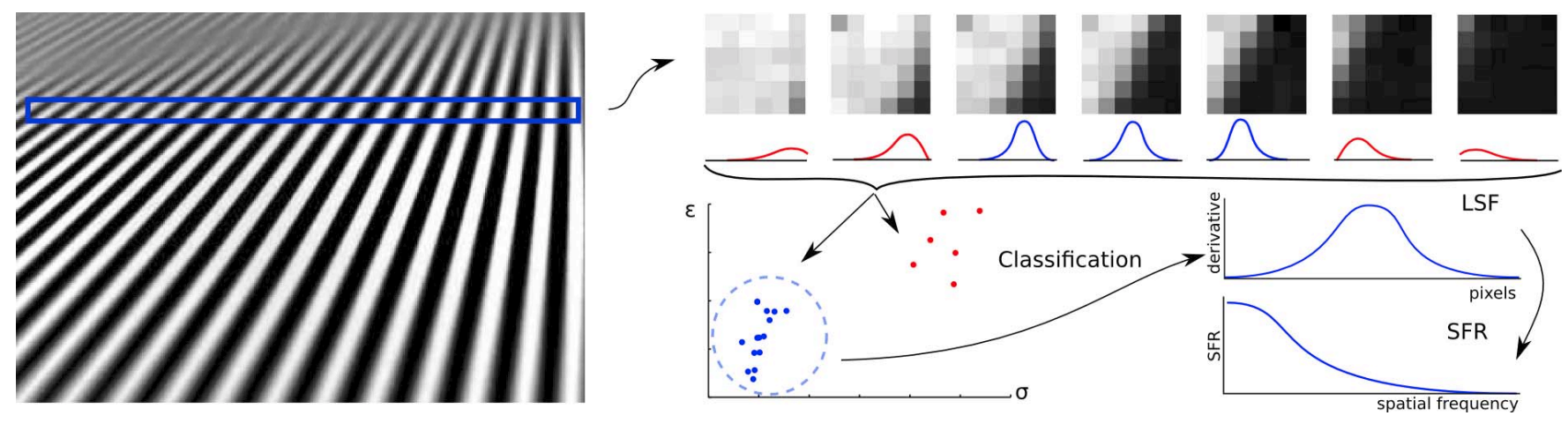

Fig. 2: SFR measurement workflow. Each row of the acquired light field corresponds to a different depth on the observed target. For each row, our method starts by selecting which microimages are valid for line spread function (LSF) estimation. The SFR is then computed by taking the modulus of the Fourier transform of the average LSF.

in front of the sensor: the main lens and the microlens array. The SFR of such cameras is subject to the interaction between those two and varies according to the depth of the scene. Consequently, the SFR also varies across the acquired image. This particular characteristic makes traditional methods not applicable anymore. In this paper, we thus propose a method to measure the SFR of a microlens-based light field camera across multiple depths using a 3-dimensional target and a single image acquisition. As seen in Fig. 1, the resolving power varies across the image and is the lowest at the focal point $[3,4]$. We conducted experiments with the Lytro and obtained results that are consistent with the theoretical bounds. Our method is straightforward to implement and reproducible for different camera parameters.

\section{RELATED WORK}

In recent literature, researchers proposed theoretical SFR measures for light field cameras by analyzing the geometry of the captured light field. $\mathrm{Ng}$ [1] derived the output resolution of photographs from the theory behind his refocusing algorithm while Lumsdaine et al. [5] studied the sampling pattern of different microlens-based designs by comparing their theoretical resolution floor. Our method is independent of image rendering algorithms and provides a practical approach to measure the resolving power of the optics/sensor combination.

SFR measurement methods for conventional cameras use different targets, such as a slanted edge [2], the Siemens star [6], or the dead leaves target [7]. The slanted edge measures the edge response of the camera with an oversampled step function. This target is appealing for our application because it can be used even when only a limited number of pixels is available, as is the case for each microimage in light field cameras. The other targets provide more comprehensive measurements but are not suitable for low resolution images, as they require a larger sensor area. Using those targets with low resolution images can produce results affected by aliasing.

\section{SFR MEASUREMENT}

We describe here the proposed workflow to measure the SFR of a microlens-based light field camera from a captured raw light field. The workflow is illustrated in Figure 2 and summarized in Algorithm 1.

Input: Raw light field captured from target

Output: SFR for each depth of the target

Pre-processing (linearization, devignetting);

Derive slanted edge angle using Hough transform;

for all microimages do

Fit Gaussian function to line spread function (LSF); end

for all rows of microimages do

Classify microimages by Gaussian fit;

Combine LSF and compute SFR;

end

Algorithm 1: High-level description of the proposed SFR measurement method.

\subsection{Target setup and pre-processing}

The camera setup described in Figure 3 shows how the target is positioned in space with respect to the camera.The target itself is made of a set of black lines printed over a white background on a planar surface. The camera is rotated and tilted with respect to the target so that slanted edges are captured at different depths. Those slanted edges are observed by the microlenses and projected on the sensor as microimages, forming the acquired raw light field. Each row of the light field corresponds to a single depths on the target. The light field is first devignetted by dividing the input image by a normalized calibration image that represents the light attenuation at each pixel due to vignetting. The light field is then preprocessed to linearize the digital output level according to the input luminance using the sensor's opto-electronic conversion function (OECF) [8], similarly to traditional SFR measurement. 


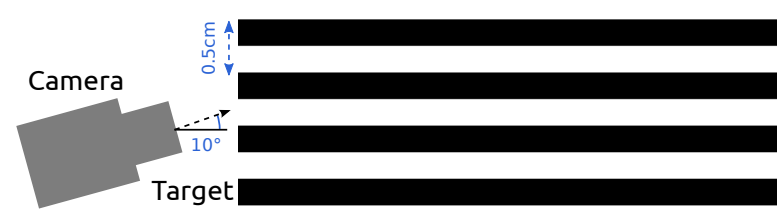

(a) Top view

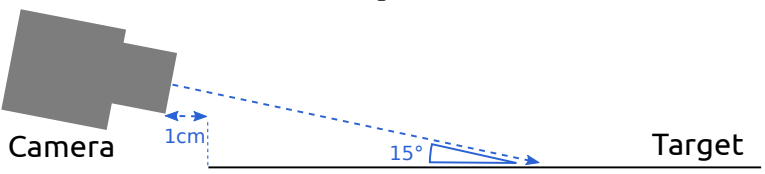

(b) Side view

Fig. 3: Target capture setup. The camera is tilted vertically and rotated horizontally with respect to the target in order to capture slanted edges at different depths.

\subsection{Microimages analysis and classification}

The angle of the slanted edges changes throughout the image because of perspective distortion. It is therefore unknown and needs to be retrieved for each microimage. The angles are found using the Hough transform of the rendered image and assigned, respectively, to each microimage based on its spatial position. Then, the line spread function (LSF) of the microimage is computed by integrating the pixel rows along this angle. The discrete derivative of the resulting edge spread function (ESF) is computed as suggested in [2, 9].

For a given row of the acquired light field, not all microimages possess a well-defined edge that can be used for SFR measurements. Consequently, to determine which microimages are usable, we need to classify them into two groups based on whether they represent a valid LSF or not. For this purpose, a Gaussian function is fitted to the LSF obtained for each microimage. Based on the spread $\sigma$ and the fitting error $\epsilon$ of the Gaussian fit, the microimages are clustered within each row of the light field and labelled as valid when they belong to the cluster with the smallest $\epsilon$. Clustering is performed using an Expectation-Maximization (EM) algorithm robust to outliers to find a Gaussian Mixture Model fitting the data. When the two clusters overlap, the EM algorithm does not converge as the camera reached its resolution floor and the blur radius is maximal.

In the next step, only the valid microimages are taken into account for SFR estimation. The classification is done rowwise as it matches the depth change of our target during the acquisition.

\subsection{SFR computation}

For each row of the light field, the LSF of the valid microimages are averaged and the SFR is computed as the modulus of the LSF's in the Fourier domain. A mathematical representation of the operation is shown in Equation 1, where $\mathcal{V}_{r}$ refers to the set of valid microimages at row $r, \mathcal{F}$ is the Fourier transform operator and $\omega$ is the frequency in cycles/pixel.

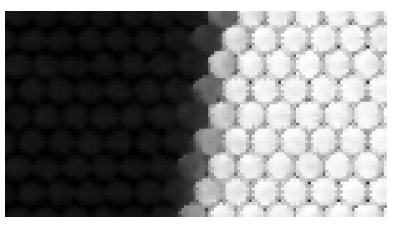

(a) At the focal point, the blur is maximal and no edge is present in the microimages.

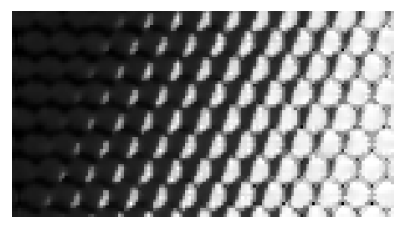

(b) Away from the focal point, multiple microlenses capture a slanted edge for which the SFR is computable.

Fig. 4: Slanted edges in microimages.

$$
\operatorname{SFR}(\omega, r)=\left|\mathcal{F}\left(\frac{1}{\left|\mathcal{V}_{r}\right|} \sum_{i \in \mathcal{V}_{r}} L S F_{i}(x)\right)\right|
$$

For the rows corresponding to the depth of the focal plane of the main lens, the blur radius is maximal and no edge is present in the microimages (see Figure 4). In this special case, the SFR cannot be estimated using microimages. This case represent the resolution floor of the camera for which the SFR can be measured from the spatial sampling of the microlens array (Figure 4a).

\section{EXPERIMENTS \& ANALYSIS}

We conducted experiments with the first generation Lytro camera [10]. The results in Figure 6a represent the SFR for a range of depths. The blue curve shows the average resolving power at $50 \%$ modulation, and the green curve shows the normalized frequency at $10 \%$ modulation, representing the limiting resolution value consistent with the Rayleigh criterion $[11,12]$. The image row number on the horizontal axis is inversely proportional to the relative depth of the target with respect to the camera. The shaded area around the curves represent the standard deviation resulting from averaging the LSFs. Figure $6 \mathrm{~b}$ shows the number of slanted edges used for each row. The values are proportional to the radius of the blur projected by the main lens on the microlens array.

The frequency values for the limiting resolving power (10\% modulation) show that the camera does not achieve Nyquist frequency (shown in red at 0.5 cycles/px). Thus, the camera does not take advantage of the full resolution of the sensor, and the highest frequencies of the observed scene will not be represented in the light field.

The shape of the SFR across the scene depth is consistent with the resolution gap identified in $[3,4]$ where the resolving power drops exactly at the focal point. The ratio between the maximum and minimum limiting frequencies is approximately 10 , which is consistent with the different sampling periods between the microlenses and the pixels in the Lytro camera design.

Figure 5 shows three measurements where the focal plane is shifted away from the camera position, all other camera parameters remaining constant. The results are coherent as the shape of the curves is stable across the three plots, thus 


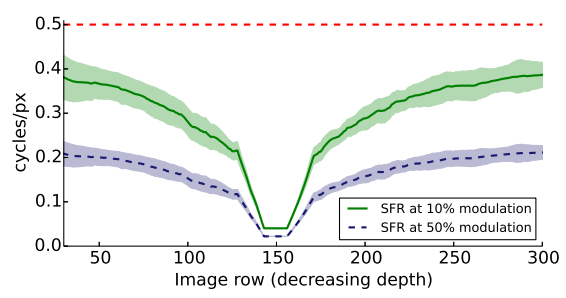

(a) Focal point at row 150

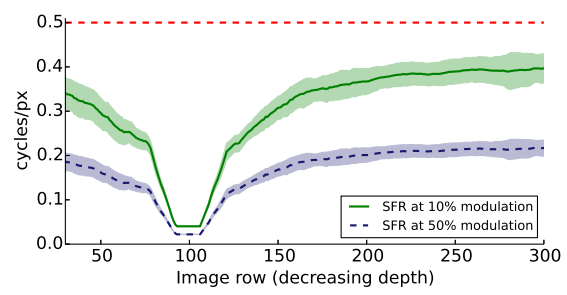

(b) Focal point at row 100

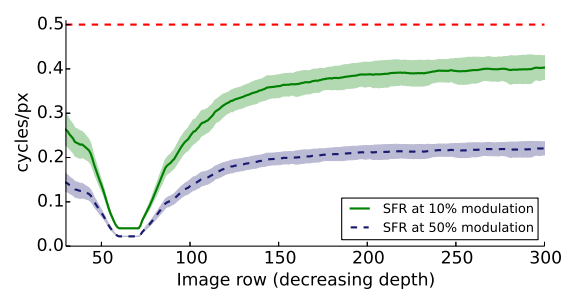

(c) Focal point at row 65

Fig. 5: SFR measurement for different focal points. As the focal point is shifted further from the camera (i.e. towards the top of the target), the depth of the resolution floor follows its location.

confirming the reproducibility of the proposed measurement method.

\section{DISCUSSION}

The algorithm presented does not depend on parameters, however modifying the target-camera setup can affect the results to a certain extent. The vertical tilt angle of the camera delimits the depth range that will be sampled and the horizontal rotation angle of the target defines the amount of slant of the edges. If the edges are not slanted enough, some microimages may contain straight edges that are unusable for SFR measurement as the edge is not oversampled by the sensor and the measurement is subject to aliasing. Vignetting of the light field (Section 3.1) may produce inexact SFR measurement as well, as it causes unequal light distribution over the microimage and reduces the sharpness of the edge.

Our motivation for using the slanted edge method is due to the low resolution of the microimages. Its simplicity comes at the cost of retrieving only a 1D slice of the PSF.

The accuracy of the results can be improved with an increased number of usable microimages, for example by capturing the target multiple times and shifting the camera horizontally for each exposure. Also, the lines on the target could be designed in such a way that it anamorphically compensate for perspective distortion, thus eliminating the angle detection step. In [13], Williams et al. analyze the reliability of modifications of the slanted edge method, some of which could be taken into consideration to increase accuracy when applied to our method.

By considering the resolution of each microlens independently, this method cannot estimate the periodical dip in resolution where information is lost due to the overlapping of light field samples [14]. To overcome this issue, the disparity between the slanted edge locations in neighboring microimages should be taken into account.

The method presented here was designed specifically for microlens-based light field cameras, such as the Lytro [10] or the Raytrix [15]. However there are different designs such as camera arrays [16] for which a comparable method can be developed to compare the performance of the different designs.

The MATLAB code used for these experiments is provided at http://ivrg.epfl.ch/research/sfr.

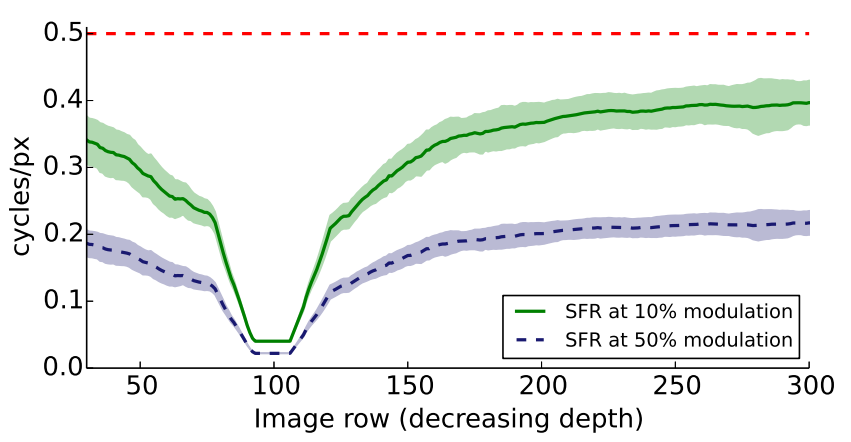

(a) Focal point at row 100

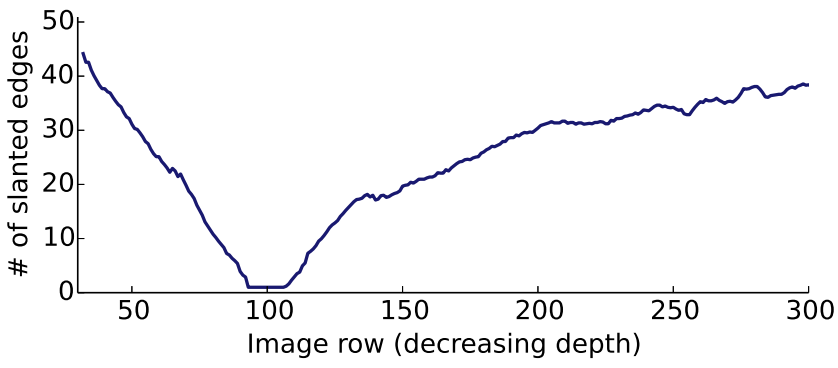

(b) Number of slanted edges used for SFR measurement

Fig. 6: SFR measurement results. Figure 6a: the frequencies at $10 \%$ and $50 \%$ SFR over a range of depths. The shaded area is the standard deviation from the LSF averaging. The red line indicates the Nyquist frequency. Figure 6b: the number of usable slanted edges is proportional to the main lens blur radius.

\section{CONCLUSION}

In this paper, we developed a method for measuring the SFR of a microlens-based light field camera. We used a 3dimensional target to capture the evolution of the SFR across different depths and through the resolution floor of the camera. We found that the experimental results obtained with our technique were qualitatively consistent with the theoretical results derived in [4]. Additionally, the method is not dependent on image rendering algorithms as we measure the camera's performance directly from the acquired raw light field. 


\section{REFERENCES}

[1] Ren Ng, Digital light field photography, Ph.D. thesis, Stanford University, 2006.

[2] ISO12233, "ISO 12233:2000: Photography-Electronic Still-Picture Cameras-Resolution Measurements," 2000.

[3] Todor Georgiev and Andrew Lumsdaine, "Depth of field in plenoptic cameras," Eurographics, pp. 5-8, 2009.

[4] Tom E. Bishop, Sara Zanetti, and Paolo Favaro, "Light field superresolution," IEEE International Conference on Computational Photography (ICCP), pp. 1-9, 2009.

[5] Andrew Lumsdaine, Todor Georgiev, and Georgi Chunev, "Spatial analysis of discrete plenoptic sampling," IS\&T/SPIE Electronic Imaging, vol. 8299, pp. 829909, 2012.

[6] Christian Loebich and Dietmar Wueller, "Digital camera resolution measurement using sinusoidal Siemens stars," IS\&T/SPIE Electronic Imaging, vol. 6502, pp. 65020N-1-65020N-11, 2007.

[7] Georges Matheron, Random sets and integral geometry, Wiley New York, 1975.

[8] ISO 14524, "ISO 14524:2009 Photography - Electronic still-picture cameras - Methods for measuring opto-electronic conversion functions (OECFs), 2009.

[9] Peter D. Burns, "Slanted-edge MTF for digital camera and scanner analysis," IS\&T PICS Conference, pp. 135$138,2000$.

[10] Lytro, “www.lytro.com,” 2011.

[11] Peter D. Burns and Don Williams, "Sampling efficiency in digital camera performance standards," IS\&T/SPIE Electronic Imaging, vol. 6808, pp. 680805, 2008.

[12] Max Born and Emil Wolf, Principles of Optics, pp. 333335, Pergamon, Oxford, UK, 6th edition, 1980.

[13] Don Williams and Peter D. Burns, "Evolution of slanted edge gradient SFR measurement," IS\&T/SPIE Electronic Imaging, vol. 9016, pp. 901605, 2014.

[14] Tom E. Bishop and Paolo Favaro, "The light field camera: Extended depth of field, aliasing, and superresolution," Pattern Analysis and Machine Intelligence, IEEE Transactions on, vol. 34, no. 5, pp. 972-986, 2012.

[15] Raytrix, "www.raytrix.de," 2010.
[16] Kartik Venkataraman, Dan Lelescu, Jacques Duparr, Andrew Mcmahon, Gabriel Molina, Priyam Chatterjee, and Robert Mullis, "PiCam : An Ultra-Thin High Performance Monolithic Camera Array," ACM Transactions on Graphics (TOG), vol. 32, no. 6, pp. 166, 2013. 